\title{
DESENVOLVIMENTO DE UM VEÍCULO ELÉTRICO DE EFICIÊNCIA ENERGÉTICA
}

\author{
Anthony Soares de Alencar ${ }^{1}$, Keyll Carlos Ribeiro Martins ${ }^{2}$, Evandro Martins Araujo Filho ${ }^{3}$, \\ Gleydson Hiago Sousa Oliveira ${ }^{4}$, Kaio Lourenço Teixeira Barbosa ${ }^{5}$, Marcelo Portela do \\ Carmo Morais ${ }^{6}$ \\ 1,2,3,4,5 e 6 Instituto Federal de Educação, Ciência e Tecnologia do Maranhão \\ E-mails: thony_ma@hotmail.com, kmartins@ifma.edu.br, evandro2.0@hotmail.com, \\ gleydson.sousa3@gmail.com, kaiobrbs@gmail.com, mpcmorais1@ hotmail.com
}

\section{RESUMO}

Uma das principais questões discutidas em diversos países é a situação da sociedade perante o problema ambiental de alcance global, chamado de efeito estufa. A constante elevação dos termômetros da terra nos leva a previsões catastróficas para um futuro não tão distante. A emissão de gases poluentes como o dióxido de carbono e o metano, são gerados principalmente pela queima de combustíveis fósseis que estão diariamente abastecendo milhões de veículos no mundo inteiro. $\mathrm{O}$ acúmulo desses gases nas camadas da atmosfera é chamado de efeito estufa. Os veículos elétricos, por possuírem emissão zero e baixo ruído, são os mais promissores para substituir os atuais veículos compostos por motores à combustão interna. O principal objetivo deste projeto é o desenvolvimento de um protótipo veicular movido por um motor elétrico a fim de obter eficiência energética, o que implica em grande autonomia, alta eficiência do conjunto motor-transmissão, e zero em emissões de gases de efeito estufa. Com o objetivo de obter valores de eficiência energética, todo o projeto, fabricação e testes do veículo estão voltados para este objetivo. O projeto propõe soluções construtivas na área da engenharia como motores de corrente continua com comutação eletrônica e transmissão simples para a tração do veículo, banco de baterias de íons de lítio e um gerenciamento eficiente e seguro de energia utilizando-se de microcontroladores, além de um projeto estrutural de chassi leve e resistente em alumínio. Todos os componentes estão interligados compondo um veículo do tipo triciclo e monoposto elétrico de eficiência energética.

\section{INTRODUÇÃO}

Mobilidade é um importante fator para muitas atividades econômicas e privadas, sendo crucial em nossas vidas. Em muitos países industrializados, a maior parte da demanda por mobilidade está no tráfego rodoviário onde veículos trafegam em grandes quantidades por redes de rodovias permitindo um grande grau de liberdade individual. No entanto, está mobilidade nos leva a substanciais problemas ambientais.

No brasil, de acordo com o Segundo Inventário Nacional, indica que o setor de transporte, sobretudo o transporte rodoviário, tem sido o principal responsável pela elevação das emissões de $\mathrm{CO} 2$ proveniente de combustíveis fósseis [1]. Devido a esse panorama, tem surgido e se consolidado programas de incentivo ao desenvolvimento de veículos livres de emissões de poluentes e mais eficientes, em particular a motorização elétrica é a mais promissora destas 
tecnologias para substituir o atual motor a combustão, permitindo carros, metros e ônibus totalmente elétricos com autonomia e eficiência.

Um veículo elétrico (EV) tem-se uma enorme vantagem inicial sobre veículos com motores a combustão interna (MCI): Veículos MCI geralmente tem-se uma eficiência em cerca de 20\% 40\%, o que significa que a maior parte do conteúdo energético de seu combustível é desperdiçado, contra, os veículos elétricos, que em geral, possuem eficiência acima de $80 \%$ [2].

\section{MATERIAL E METODOLOGIA}

Para a realização do presente trabalho, foi realizado ampla revisão bibliográfica do assunto, em especial com foco em informações relacionados à dinâmica veicular e eletroeletrônica automotiva. Na etapa posterior, a proposta de concepção do veículo foi assim então dividida em seis sistemas: Estrutura, motor e transmissão, direção, freio, bateria e eletroeletrônica.

\subsection{Estrutura}

Para a estrutura do veículo, foi priorizado a concepção de um veículo leve, seguro e ergonômico do tipo monoposto, com o objetivo de proteger o ocupante de possíveis impactos e abrigar todos os componentes para o funcionamento do veículo.

No projeto foi estabelecido que o material a ser utilizado na construção do chassi seria tubos de liga de alumínio 6061 T6 com as propriedades químicas e mecânicas listadas na tabela 1 .

Tabela 1: Propriedades químicas e mecânicas do alumínio 6061.

\begin{tabular}{|c|c|c|}
\hline \multicolumn{3}{|l|}{ Propriedades Químicas (\%) } \\
\hline $\mathrm{Si}$ & \multicolumn{2}{|c|}{$0,40-0,80$} \\
\hline $\mathrm{Fe}$ & \multicolumn{2}{|c|}{0,70} \\
\hline $\mathrm{Cu}$ & \multicolumn{2}{|c|}{$0,15-0,40$} \\
\hline $\mathrm{Mn}$ & \multicolumn{2}{|c|}{0,15} \\
\hline $\mathrm{Mg}$ & \multicolumn{2}{|c|}{$0,80-1,20$} \\
\hline $\mathrm{Zn}$ & \multicolumn{2}{|c|}{0,25} \\
\hline $\mathrm{Ti}$ & \multicolumn{2}{|c|}{0,15} \\
\hline $\mathrm{Al}$ & \multicolumn{2}{|c|}{$96,50-99,00$} \\
\hline Outros & \multicolumn{2}{|c|}{$0,05-0,15$} \\
\hline Propriedades Mecânicas & Min & Max \\
\hline Limites de Resistência à Tração (Mpa) & 290 & 350 \\
\hline Limite de Escoamento (Mpa) & 255 & 330 \\
\hline Alongamento mínimo em $\%(50 \mathrm{~mm})(1,6 \mathrm{~mm}$ de espessura $)$ & \multicolumn{2}{|c|}{$8-16$} \\
\hline Dureza Brinell & \multicolumn{2}{|c|}{$95-110$} \\
\hline
\end{tabular}




\subsection{Motor e Transmissão}

O motor elétrico usado na propulsão possui $250 \mathrm{~W}$ de potência, sendo do tipo corrente contínua sem escovas, ou BLDC (Brushless DC) como visto na figura 1. Neste tipo de motor o rotor tem ímãs permanentes e o estator tem um campo rotativo eletronicamente controlado, o peso deste motor também é reduzido já que elimina-se os componentes mecânicos como as escovas[3].

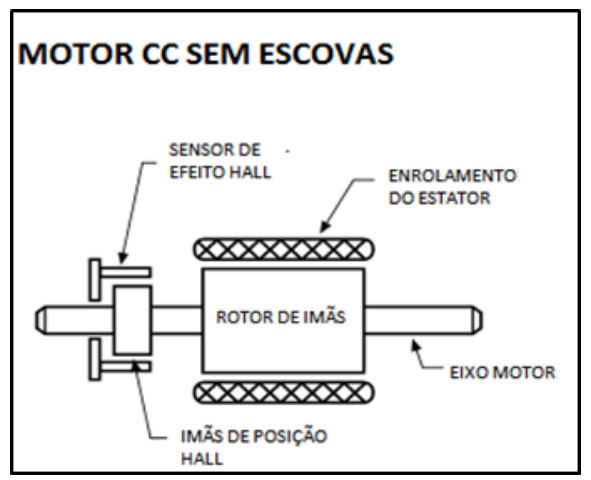

Figura 1: Esquema de motores do tipo BLDC

O sistema de transmissão escolhido é do tipo direto, com apenas uma relação de transmissão final, com redução realizada por engrenagens planetárias, sendo acopladas no próprio motor, que, por sua vez, é acoplado no aro da roda de propulsão, economizando espaço e peso. A figura 2 ilustra bem a nova configuração da transmissão proposta.

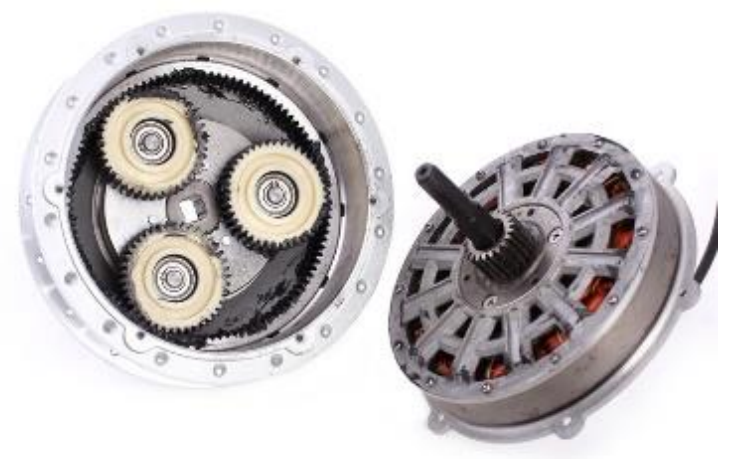

Figura 2: Sistema de transmissão por engrenagens planetárias no cubo da roda motora

\subsection{Direção}

O sistema de direção funciona a partir do mesmo princípio do sistema de direção dos carros, utilizando o princípio de Ackerman. A teoria de Ackerman surgiu devido a um pequeno problema nos sistemas de direção. Ao contornar uma curva, as rodas direcionais do veículo percorrem uma trajetória circular. Porém, essa trajetória é mais curta para a roda interna do que para a roda externa. Devido a isso, existe a necessidade de que a roda interna à curva esterce um pouco mais que a roda externa, logo surgindo a condição de Ackerman representada pela equação 1 .

$$
\cot \delta_{o}-\cot \delta_{i}=\frac{w(\text { Entre eixos do veiculo })}{l(\text { bitola das rodas dianteiras })}
$$


Em que $\boldsymbol{\delta}_{\boldsymbol{i}}$ é o ângulo de esterçamento da roda interna e $\boldsymbol{\delta}_{\boldsymbol{o}}$ é o ângulo de esterçamento da roda externa. A roda interna e externa são âmbas definidas de acordo com a origem do raio de giro criado pelo véiculo em movimento circular.

Este princípio também é utilizado no veículo e, além disso, foi dimensionado mangas de eixo para ajustar os ângulos de esterçamento das rodas, a qual estão conectadas aos terminais de direção. Esses terminais são aparafusados nas mangas de eixo e na coluna de direção, que faz a ligação entre os dois componentes por meio de braços de direção que vai, assim, transmitir a força exercida na coluna de direção para a manga de eixo da roda. O sistema todo está visualizado na figura 3(a) e figura 3(b).
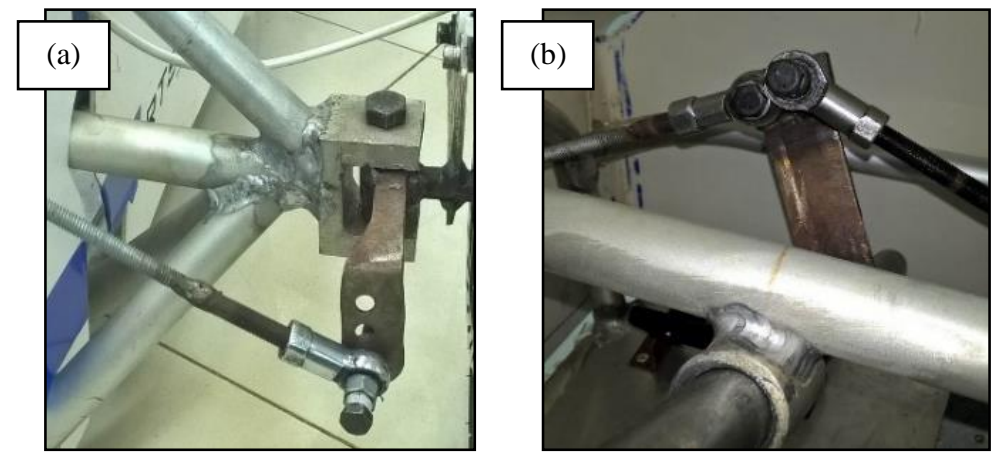

Figura 3: Sistema de Direção (a) Manga de eixo com terminal de direção; (b) Coluna de direção.

\subsection{Freio}

O sistema físico de freio do veículo proposto localiza-se nas rodas dianteiras e na roda de tração traseira, e seu funcionamento baseia-se em aplicação de força em um ponto de acionamento, com transmissão hidráulica para um outro ponto, chamado de pinça de freio, que força as pastilhas de freio a realizarem atrito sobre um disco de aço inox acoplado ao cubo da roda, diminuindo assim a rotação do veículo, como visto na figura 4.

\subsection{Bateria e Eletroeletrônica}

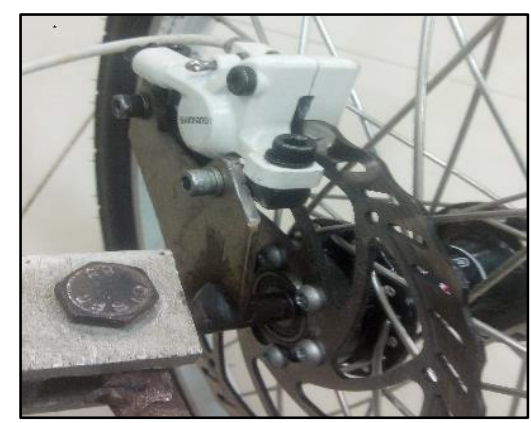

Figura 4: Sistema de frenagem

Uma das grandes preocupações na concepção de um veículo elétrico é a definição do acumulador de energia ideal para proporcionar a potência necessária para o motor, no caso do veículo proposto, é utilizado um banco de baterias composta por células de Óxido Lítio-Cobalto (LiCo2), devido sua característica de armazenar o dobro de energia que uma bateria convencional, e estarem livres do efeito memória, característica benéfica para a comodidade e portabilidade do sistema. Na figura 5 temos a bateria utilizada no protótipo. 


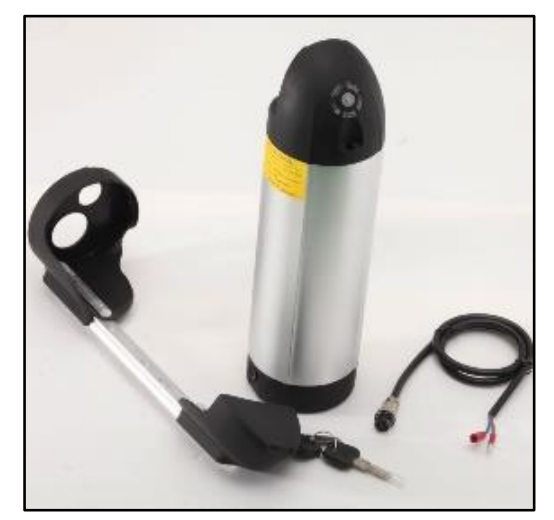

Figura 5: Bateria de lítio do veículo

Para controlar o motor BLDC, é necessário um circuito de potência, como visto na figura 6, composto por drivers, transistores do tipo mosfet, capacitores, diodos e micro controladores capazes de processar os sensores localizados no motor para que ocorra a correta sincronização na condução das correntes das fases do motor [3].

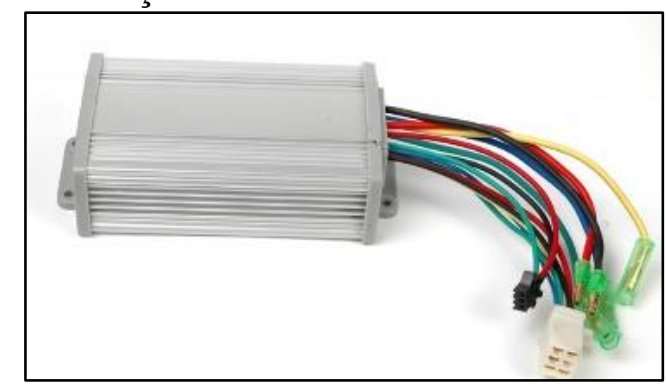

Figura 6: Controlador eletrônico do motor elétrico do veículo

\section{RESULTADOS}

\subsection{Resultados Estruturais}

Para obter os resultados estruturais do projeto foi necessário a utilização do software de desenho assistido por computador SolidWorks. Para a análise estática do chassi a simulação foi realizada utilizando tubos de liga de alumínio 6061 , de $25 \mathrm{~mm}$ diâmetro externo e 1,2 mm de espessura, para confirmar a segurança da estrutura e proceder com a fabricação da mesma. No projeto descrito foi estabelecido o carregamento com distribuição de $800 \mathrm{~N}$, nas vigas inferiores, onde o condutor estará apoiado, distribuídas na parte inferior do veículo, e posteriormente foi analisado o critério de falha de Von Mises, visto na figura 7 e o fator de segurança figura 8. 


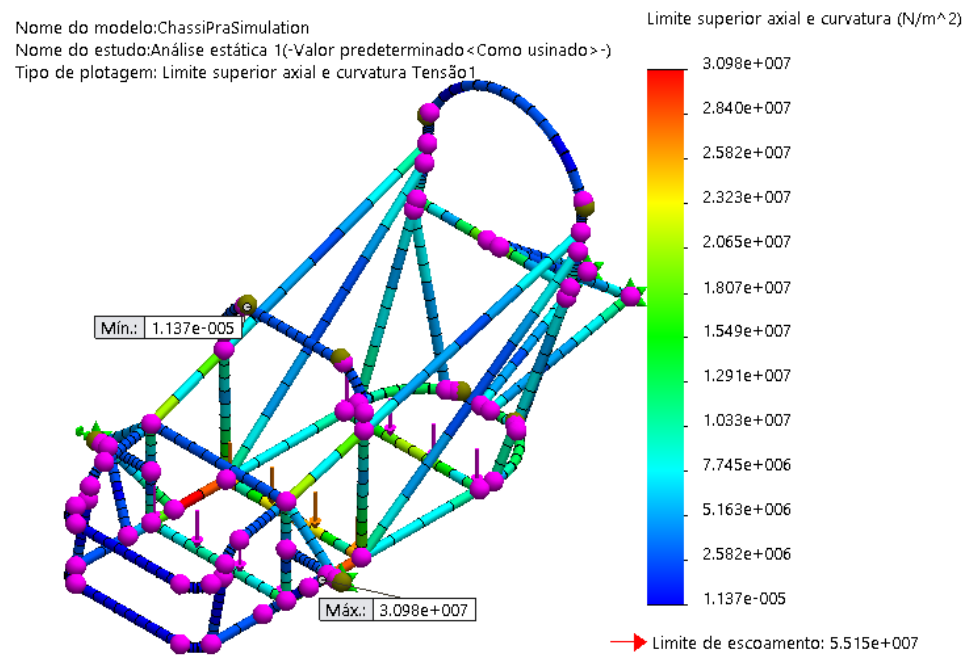

Figura 7: Simulação do Critério de falha de Von Mises

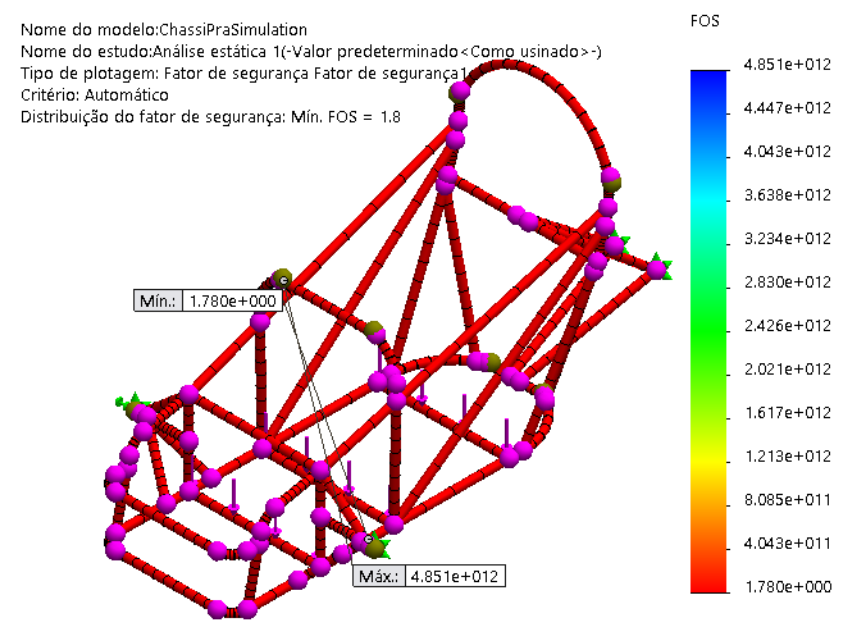

Figura 8: Simulação do fator de segurança do chassi do veículo.

A figura acima representa o fator de segurança do chassi do veículo. O valor mínimo encontrado foi de 1.78 e o máximo de $4 * 10^{12}$, o que garante um fator de segurança acima de 1 em toda a sua estrutura, concluindo que a estrutura proposta está corretamente dimensionada para o veículo.

\subsection{Análise do sistema de direção}

Para o início da análise do sistema de direção, fixou-se um raio de giro mínimo do veículo em 8 metros. A equação 4 demonstra o cálculo do raio de giro do veículo.

$$
\text { Raio de giro }=\frac{\text { Bitola dianteira }(w)}{2}+\frac{\text { Entre eixos }(l)}{\sin (\text { angulo médio }(\delta))}
$$

Em que os parâmetros do veículo são:

Raio de giro $=8 \mathrm{~m}$.

Entre eixos $=126 \mathrm{~cm}$

Bitola dianteira $=96 \mathrm{~cm}$ 
Resolvendo a Eq. (2) obtemos que o ângulo de mínimo de esterçamento para o veículo proposto é de $\boldsymbol{\delta}>9.645^{\circ}$ para um raio de giro de no mínimo 8 metros.

Para estar de acordo com a geometria de Ackerman, utilizaremos um raio de esterçamento da roda externa igual ao ângulo mínimo de esterçamento do veículo, com $\boldsymbol{\delta} \boldsymbol{o}=\mathbf{9 . 6 4 5 ^ { \circ }}$, $\log \mathrm{o}$, retornando a Eq. (1) temos:

$$
\cot (9.645)-\cot \delta_{i}=\frac{0.96(w)}{0.126(l)}
$$

Logo obtemos um $\boldsymbol{\delta} \boldsymbol{i}=\mathbf{1 1 . 0 4 5}^{\circ}$, nestas condições, assumimos então analiticamente que o ângulo ideal de esterçamento externo deverá ser $\boldsymbol{\delta} \boldsymbol{o}=\mathbf{9 . 6 4 5 ^ { \circ }}$, e o ângulo ideal de esterçamento interno $\boldsymbol{\delta} \boldsymbol{i}=\mathbf{1 1 . 0 4 5}$.

A simulação em CAD utilizando o software Solidworks reproduz os ângulos $\boldsymbol{\delta} \boldsymbol{o} \boldsymbol{e} \boldsymbol{\delta} \boldsymbol{i}$ no sistema de direção do veículo, dentro das margens aceitas para a geometria de Ackerman. Figura 9.

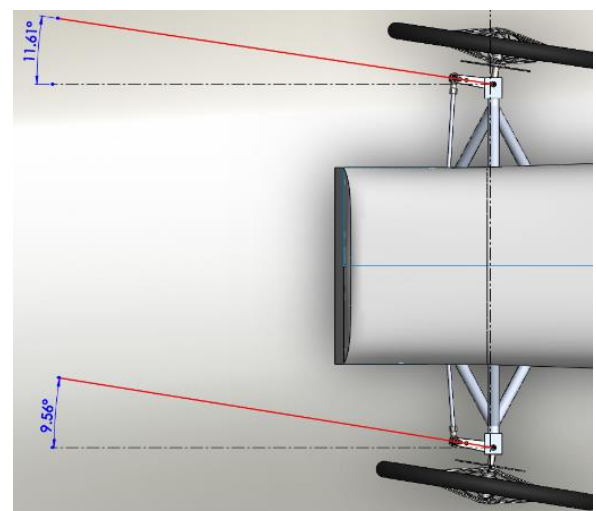

Figura 9: Simulação em CAD dos ângulos de direção do veículo

\subsection{Análise do protótipo}

A configuração final do protótipo com todos os sistemas envolvidos na dinâmica veicular, além dos componentes de eletroeletrônica, são mostrados na figura 10 , junto com o esboço do protótipo na figura 11. Mais detalhes do veículo também está especificado na ficha técnica da tabela 2.

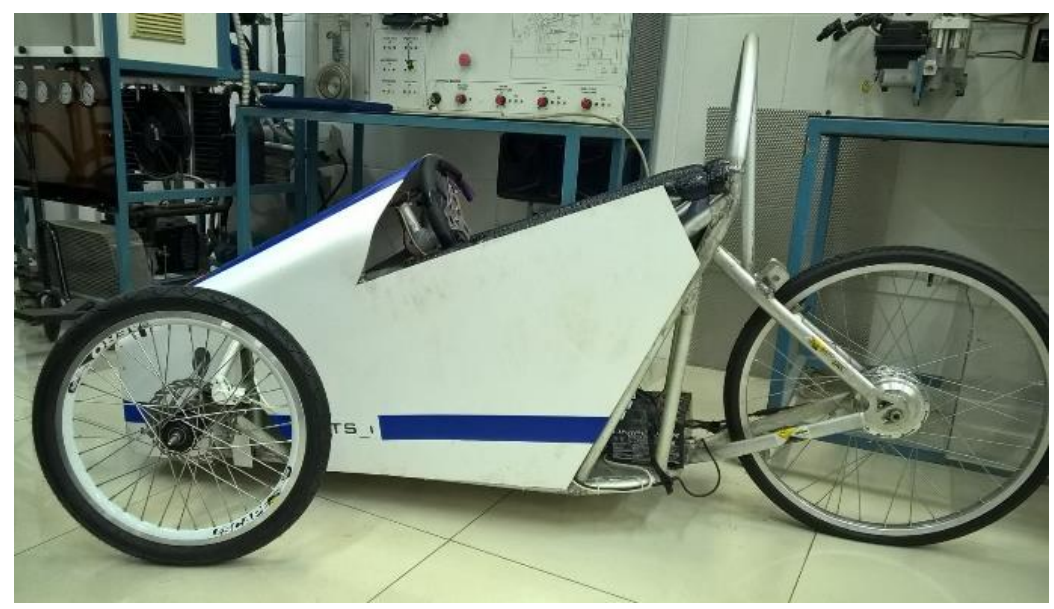

Figura 10: Protótipo final construído 


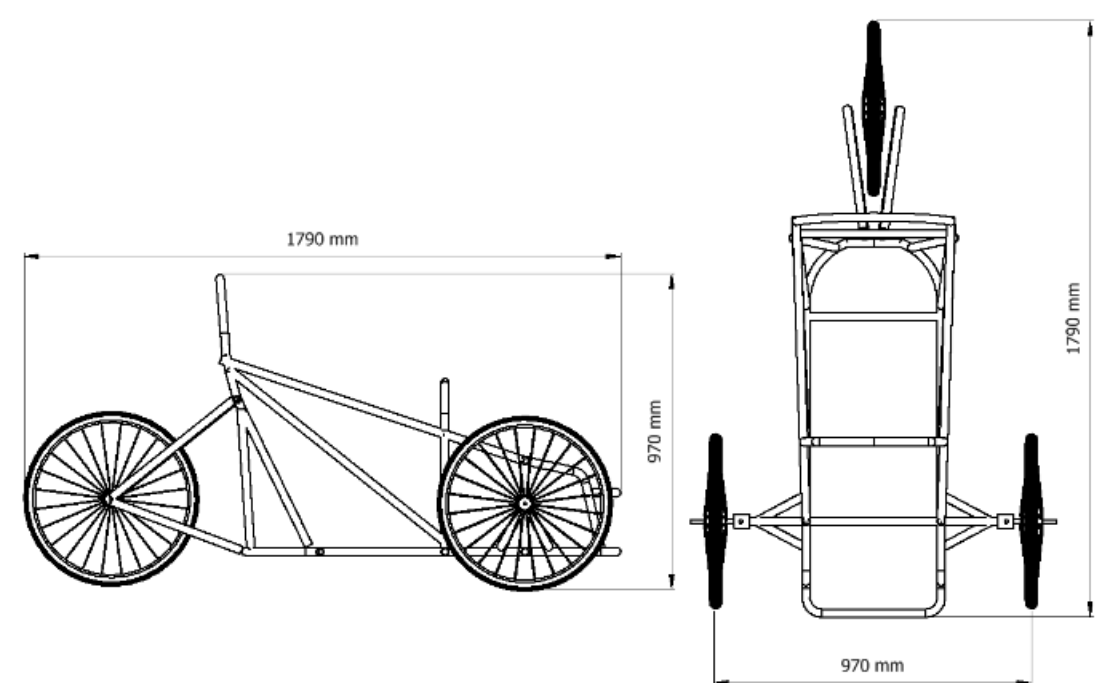

Figura 11: Esboço do protótipo

Tabela 2: Ficha técnica do veículo elétrico proposto

\begin{tabular}{|l|l|l|}
\hline $\mathbf{N}$ & ITENS & ESPECIFICAÇÕES \\
\hline $\mathbf{1}$ & Largura do Veículo & $\begin{array}{l}600 \mathrm{~mm} \text { *Estrutura e } 970 \mathrm{~mm} \text { *Bitola } \\
\text { dianteira }\end{array}$ \\
\hline $\mathbf{2}$ & Comprimento do Veículo & $1790 \mathrm{~mm}$ \\
\hline $\mathbf{3}$ & Altura do Veículo & $970 \mathrm{~mm}$ \\
\hline $\mathbf{4}$ & Peso do Veículo & $35 \mathrm{Kg}$ \\
\hline $\mathbf{5}$ & Propulsão & Motor elétrico BLDC, 36v \\
\hline $\mathbf{6}$ & Potência máxima & $250 \mathrm{Watts}(0.3 \mathrm{hp})$ \\
\hline $\mathbf{7}$ & Torque máximo & $17,4 \mathrm{~N} / \mathrm{m}$ \\
\hline $\mathbf{8}$ & Velocidade máxima & $30 \mathrm{~km} / \mathrm{h}$ *limitado eletronicamente \\
\hline $\mathbf{9}$ & Bateria & Banco de baterias de Ions de Lítio 36v 11 ah \\
\hline
\end{tabular}

\section{CONCLUSÃO}

O presente projeto encontra-se finalizado no que tange o desenvolvimento, construção e análise do correto funcionamento de um veículo de eficiência puramente elétrico. Com ênfase nos resultados das simulações estruturais e cálculos de dinâmica veicular, atingindo bons resultados práticos, analíticos e simulados. Espera-se que com os resultados deste trabalho possa proporcionar boas colocações na competição Shell Eco-Marathon Brasil em 2017, sendo este protótipo veícular participante na categoria de veículos elétricos representando o Instituto Federal do Maranhão.

\section{AGRADECIMENTOS}

Ao meu amigo e orientador Dr. Keyll Carlos Ribeiro Martins, por ter me auxiliado com suas precisas e incisivas pontuações. 
Ao Departamento de Mecânica e Materiais do IFMA, por fornecer meios de realização da pesquisa. E a todas as outras pessoas que direta ou indiretamente colaboraram com a realização deste trabalho.

\section{REFERÊNCIAS}

[1] MINISTÉRIO DA CIÊNCIA, TECNOLOGIA E INOVAÇÃO (MCTI). "Segunda Comunicação Nacional do Brasil à Convenção-Quadro das Nações Unidas Sobre Mudanças do Clima \& Segundo Inventário Brasileiro das Emissões e Remoções Antrópicas de Gases de Efeito Estufa", Vol. I. Brasília (DF), 2010.

[2] G. Tobias; J. Anders. "Comparison between Battery Electric Vehicles and Internal Combustion Engine Vehicles fueled by Electrofuels", Chalmers University of Technology, Sweden, 2015.

[3] R. Krishnan. "Permanent Magnet Synchronous and Brushless DC Motor Drives: Theory, Operation, Performance, Modelling, Simulation, Analysis and Design, Part 3: Permanent Magnet Brushless DC Machines and Their Control.” Virginia Tech, Blacksburg; 